\title{
The changes in the panel of circulating cytokines in patients with type 2 diabetes and chronic kidney disease
}

\author{
Anton Korbut \\ Laboratory of Endocrinology \\ Research Institute of Clinical and \\ Experimental Lymphology - Branch of the \\ Institute of Cytology and Genetics, \\ Siberian Branch of Russian Academy of \\ Sciences (RICEL - Branch of IC\&G SB \\ RAS) \\ Novosibirsk, Russia \\ ORCID: 0000-0003-3502-5892
}

\author{
Nikolaj Orlov \\ Laboratory of Clinical Immunogenetics \\ Research Institute of Clinical and \\ Experimental Lymphology - Branch of the \\ Institute of Cytology and Genetics, \\ Siberian Branch of Russian Academy of \\ Sciences (RICEL - Branch of IC\&G SB RAS) \\ Novosibirsk, Russia \\ ORCID: 0000-0002-3437-7151
}

\author{
Maksim Dashkin \\ Laboratory of Endocrinology \\ Research Institute of Clinical and \\ Experimental Lymphology - Branch of the \\ Institute of Cytology and Genetics, \\ Siberian Branch of Russian Academy of \\ Sciences (RICEL - Branch of IC\&G SB \\ RAS) \\ Novosibirsk, Russia \\ ORCID: 0000-0002-5099-5144
}

\author{
Ilya Vinogradov \\ Clinical Laboratory \\ "MBU-Technology" Ltd., \\ Novosibirsk, Russia \\ ORCID: 0000-0002-1499-0995
}

\author{
Vyacheslav Romanov \\ Clinical Laboratory \\ "MBU-Technology" Ltd., \\ Novosibirsk, Russia \\ ORCID: 0000-0003-1953-2536
}

\begin{abstract}
The study estimates the changes in the panel of circulating cytokines in patients with type 2 diabetes (T2D) and chronic kidney disease (CKD). We examined 130 patients with $T 2 D$ duration $\geq 10$ years, including 65 individuals with estimated glomerular filtration rate (eGFR) $<60 \mathrm{ml} / \mathrm{min} \times 1.73 \mathrm{~m}^{2}, 64$ patients with increased albuminuria, and 30 healthy subjects as control. Serum IL-1及, IL-1 ra, IL-2, IL-4, IL-5, IL-6, IL-7, IL-8, IL-9, IL-10, IL-12 (p70), IL-13, IL-15, IL-17A, bFGF, eotaxin,

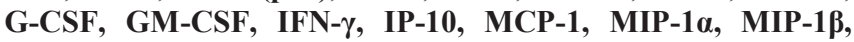
PDGF-BB, RANTES, TNF- $\alpha$, and VEGF were assessed by multiplex analysis. Serum high-sensitivity $C$-reactive protein (hsCRP), urinary nephrin and podocin, podocyte injury markers, and WFDC-2, a marker of tubulointerstitial fibrosis, were assessed by ELISA. Patients with T2D and reduced eGFR had increased levels of hsCRP, IL-1ra, IL-8, IL-13 and decreased IL-7, IL-9 and IL-15 concentrations when compared to those with preserved renal function and control (all $p<0.05$ ). Excretion of WFDC-2, but not podocyte markers, was increased in these patients $(p<0.02)$. The elevation of albuminuria was associated with enhanced excretion of nephrin and podocin $(p<0.0001)$ without difference in the levels of measured cytokines. The levels of IL-2, IL-4, IL-5, IL-17A, bFGF, G-CSF, IP-10 and MIP-1 $\alpha$ correlated negatively with eGFR; whereas WFDC-2 positively correlated with hsCRP and MIP-1 $\alpha$ and negatively correlated with IL-10 and IL-15. These results demonstrate that decreased eGFR and increased urinary excretion of WFDC-2, a marker of tubulointerstitial fibrosis, are associated with predominance of pro-inflammatory and fibrogenic molecules in the panel of circulating cytokines in T2D subjects.
\end{abstract}

Keywords - type 2 diabetes, chronic kidney disease, albuminuria, cytokines, inflammation, fibrosis.

\section{Introduction}

A number of recent studies showed that non-albuminuric chronic kidney disease (CKD) became wide-spread complication in patients with type 2 diabetes (T2D) [1,2] with higher mortality risk in some cohorts as compared to albuminuric patients [3]. It has been suggested that albuminuric and non-albuminuric CKD patterns could be different in their phenotypes and pathogenic mechanisms. The growing body of evidences indicates that chronic low-grade inflammation is involved in development of CKD in obesity [4,5] and T2D [6-10]. This study aims to estimate the differences in a panel of circulating cytokines mediating lowgrade inflammation and fibrosis in patients with T2D and different patterns of CKD.

\section{Matherials and Methods}

The single-center cross-sectional study included 130 adult male and female patients with T2D duration $\geq 10$ years. Nondiabetic CKD, end-stage renal disease, urinary tract infection, treatment with DPP-4 inhibitors, GLP-1 receptor agonists and/or SGLT-2 inhibitors for three months prior to inclusion were considered as principal exclusion criteria.

Individuals with eGFR $\geq 60 \mathrm{~mL} / \mathrm{min} \times 1.73 \mathrm{~m}^{2}$ and urinary albumin-to-creatinine ratio (UACR) $<3.0 \mathrm{mg} / \mathrm{mmol}$ were recorded as patients without chronic kidney disease (CKD) signs (CKD- group, $\mathrm{N}=33$ ). Those with eGFR $<60$ $\mathrm{mL} / \mathrm{min} \times 1.73 \mathrm{~m}^{2}$ and UACR $<3.0 \mathrm{mg} / \mathrm{mmol}$ were assigned to the non-albuminuric chronic kidney disease group (NA-CKD group, $\mathrm{N}=33$ ). Patients with eGFR $\geq 60 \mathrm{~mL} / \mathrm{min} \times 1.73 \mathrm{~m}^{2}$ and UACR $\geq 3.0 \mathrm{mg} / \mathrm{mmol}$ were defined as albuminuric with preserved renal function (A-CKD- group, $\mathrm{N}=32$ ). Individuals with eGFR $<60 \mathrm{~mL} / \mathrm{min} \times 1.73 \mathrm{~m}^{2}$ and UACR $\geq 3.0 \mathrm{mg} / \mathrm{mmol}$ comprised the albuminuric CKD group (A-CKD+ group, $\mathrm{N}=32$ ). Thirty healthy normoglycemic subjects (11 men and 19 women) without obesity and cardiovascular disease and corresponded to participants by age were acted as control. 
Fasting serum and morning urine samples were used for laboratory investigations. Serum IL-1 $\beta$, IL-1ra, IL-2, IL-4, IL5, IL-6, IL-7, IL-8, IL-9, IL-10, IL-12 (p70), IL-13, IL-15, IL17A, bFGF, eotaxin, G-CSF, GM-CSF, IFN- $\gamma$, IP-10, MCP-1, MIP- $1 \alpha$, MIP- $1 \beta$, PDGF-BB, RANTES, TNF- $\alpha$, and VEGF were assessed by multiplex analysis. Serum high-sensitivity C-reactive protein (hsCRP), urinary nephrin and podocin, as podocyte injury markers, and urinary WAP four-disulfide core domain protein 2 (WFDC-2), a marker of tubulointerstitial fibrosis, were assessed by ELISA. The results were adjusted to the urinary creatinine levels.

\section{Results}

Urinary nephrin and podocin excretions were increased significantly in all diabetic groups (all $p \leq 0.02$ ). Both markers were higher in A-CKD- and A-CKD+ groups when compared to $\mathrm{CKD}$ - and NA-CKD subjects (all $\mathrm{p} \leq 0.04$ ). Patients from CKD- and NA-CKD as well as patients from A-CKD- and A$\mathrm{CKD}+$ groups did not differ by nephrin and podocin excretion. Urinary WFDC2 concentrations were markedly higher in men than in women $(p<0.000001)$. Men from CKD-, NA-CKD and $\mathrm{A}-\mathrm{CKD}+$ groups demonstrated increased WFDC-2 excretion compared to control ones ( $\mathrm{p}=0.04, \mathrm{p}=0.01$ and $\mathrm{p}=0.009$, respectively), without significant differences between diabetic groups. Women with NA-CKD or A-CKD+ had elevated excretion of WFDC-2 as compared to control and CKDwomen (all $\mathrm{p} \leq 0.01)$.

Patients with T2D had higher serum concentrations of CRP when compared to non-diabetic control $(\mathrm{p}=0.009)$; the changes were more prominent in A-CKD+ group ( $\mathrm{p}=0.03$ vs. control). Patients with T2D demonstrated elevated serum levels of IL-1ra, IL-2, IL-4, IL-13, IL-17A, eotaxin, bFGF, GCSF, IP-10 and MIP- $1 \alpha$ and reduced serum levels of IL-9, IL12 , IL-15, GM-CSF, INF- $\gamma$, and VEGF as compared to control $($ all $\mathrm{p}<0.05)$.

Patients with NA-CKD had higher levels of IL-2, IL-4, IL5 , IL-8, IL-17A, bFGF, G-CSF, MIP- $1 \alpha$ and lower level of VEGF when compared to control and CKD- group (all $\mathrm{p}<0.05$ ). The A-CKD- group was characterized by increased levels of IL-4, IL-7, IL-12, IL-17, G-CSF, IP-10 and MIP-1 $\alpha$ (all $\mathrm{p}<0.05$ ). Moreover, A-CKD- patients had higher levels of IL-1ra, bFGF, GM-CSF and lower concentration of VEGF compared to control $(p<0.05)$. Patients of NA-CKD group showed higher levels of bFGF, G-CSF, GM-CSF, MIP-1 $\alpha$, IL4 , IL-8 and IL-17 when compared to patients with A-CKD+ (all $\mathrm{p}<0.05)$.

In a multiple logistic regression analysis, reduced eGFR was associated with hsCRP, IL-4, IL-10, and MIP-1 $\alpha$ levels (all $\mathrm{p}<0.05$ ). However, no associations between albuminuria and a panel of cytokines or hsCRP were found. The levels of hsCRP, bFGF, G-CSF, IP-10, MIP-1 $\alpha$, IL-2, IL-4, IL-5 and IL-17 correlated negatively with eGFR. The excretion of WFDC-2 correlated positively with MIP- $1 \alpha$ and hsCRP levels; whereas, negative correlations were found between WFDC-2 and IL-10, IL-15 and VEGF. No correlations were observed between cytokines and markers of glomerular dysfunction: UACR, nephrin, and podocin.

\section{Conclusion}

The obtained results demonstrate that both albuminuric and non-albuminuric pattern of CKD are associated with chronic low-grade inflammation in T2D patients. The decreased eGFR and increased urinary excretion of WFDC-2, a marker of tubulointerstitial fibrosis, are associated with a predominance of pro-inflammatory and fibrogenic regulators in the panel of circulating cytokines in T2D subjects.

\section{REFERENCES}

[1] F. Viazzi, G.T. Russo, A. Ceriello, P. Fioretto, C. Giorda, S. De Cosmo, R. Pontremoli. "Natural history and risk factors for diabetic kidney disease in patients with T2D: lessons from the AMD-annals", J Nephrol 2019, vol. 32(4), pp. 517-525. doi:10.1007/s40620-018-00561-3.

[2] V.V. Klimontov, A.I. Korbut. "Albuminuric and non-albuminuric patterns of chronic kidney disease in type 2 diabetes", Diabetes and Metabolic Syndrome: Clinical Research and Reviews 2019, vol. 13(1), pp. 474-479. doi: 10.1016/j.dsx.2018.11.014.

[3] G. Penno, A. Solini, E. Orsi, E. Bonora, C. Fondelli, R. Trevisan, et al. "Non-albuminuric renal impairment is a strong predictor of mortality in individuals with type 2 diabetes: the Renal Insufficiency And Cardiovascular Events (RIACE) Italian multicentre study", Diabetologia 2018, vol. 61(11), pp. 2277-2289. doi:10.1007/s00125018-4691-2.

[4] I.A. Bondar, V.V. Klimontov, A.I. Simakova. "Obesity and chronic kidney disease", Therapeutic Archive 2011, vol. 83(6), pp. 66-70.

[5] J.I. Lakkis, M.R. Weir. "Obesity and Kidney Disease", Prog Cardiovasc Dis 2018, vol. 61(2), pp. 157-167. doi:10.1016/j.pcad.2018.07.005.

[6] I.A. Bondar, V.V. Klimontov. "Immune inflammatory mechanisms in development of diabetic nephropathy", Probl. Endokrinol. 2007, vol. 53(2), pp. 34-40.

[7] V.I. Konenkov, V.V. Klimontov, N.E. Myakina, N.V. Tyan, O.N. Fazullina, V.V. Romanov. "Increased serum concentrations of inflammatory cytokines in type 2 diabetic patients with chronic kidney disease", Therapeutic Archive 2015, vol. 87(6), pp. 45-49. doi: 10.17116/terarkh201587645-49.

[8] V.V. Klimontov, N.V. Tyan, O.N. Fazullina, N.E. Myakina, A.P. Lykov, V.I. Konenkov. "Clinical and metabolic factors associated with chronic low-grade inflammation in type 2 diabetic patients", Diabetes Mellitus 2016, vol. 19(4), pp. 295-302. doi: 10.14341/DM7928.

[9] R. Pichler, M. Afkarian, B.P. Dieter, K.R. Tuttle. "Immunity and inflammation in diabetic kidney disease: translating mechanisms to biomarkers and treatment targets", Am J Physiol Renal Physiol 2017, vol. 312(4), pp. F716-F731. doi:10.1152/ajprenal.00314.2016.

[10] R.E. Pérez-Morales, M.D. Del Pino, J.M. Valdivielso, A. Ortiz, C. Mora-Fernández, J.F. Navarro-González. "Inflammation in Diabetic Kidney Disease", Nephron 2019, vol. 143(1), pp. 12-16. doi:10.1159/000493278. 